\title{
Phase-controllable nonlocal spin polarization in proximitized nanowires
}

\author{
X. P. Zhang, ${ }^{1,2,{ }^{*}}$ V. N. Golovach,,${ }^{1,2,3}$ F. Giazotto $\odot,{ }^{4}$ and F. S. Bergeret $\odot^{2,1, \dagger}$ \\ ${ }^{1}$ Donostia International Physics Center (DIPC), Manuel de Lardizabal, 4, E-20018 San Sebastian, Spain \\ ${ }^{2}$ Centro de Fisica de Materiales (CFM-MPC), Centro Mixto CSIC-UPV/EHU, E-20018 Donostia-San Sebastian, Basque Country, Spain \\ ${ }^{3}$ IKERBASQUE, Basque Foundation for Science, E-48011 Bilbao, Spain \\ ${ }^{4}$ NEST Istituto Nanoscienze-CNR and Scuola Normale Superiore, I-56127 Pisa, Italy
}

(Received 24 February 2020; revised manuscript received 7 April 2020; accepted 16 April 2020; published 5 May 2020)

\begin{abstract}
We study the magnetic and superconducting proximity effects in a semiconducting nanowire (NW) attached to superconducting leads and a ferromagnetic insulator (FI). We show that a sizable equilibrium spin polarization arises in the NW due to the interplay between the superconducting correlations and the exchange field in the FI. The resulting magnetization has a nonlocal contribution that spreads in the NW over the superconducting coherence length and is opposite in sign to the local spin polarization induced by the magnetic proximity effect in the normal state. For a Josephson-junction setup, we show that the nonlocal magnetization can be controlled by the superconducting phase bias across the junction. Our findings are relevant for the implementation of Majorana bound states in state-of-the-art hybrid structures.
\end{abstract}

DOI: 10.1103/PhysRevB.101.180502

Semiconducting nanowires (NWs) in proximity to superconductors (SCs) are central to the creation of a topologically nontrivial superconducting state, which manifests itself through Majorana zero modes at the edges of the NW [1-13]. The basic ingredients needed for the topological phase are the spin-orbit interaction (SOI), superconducting correlations, and Zeeman splitting [14-20]. Whereas SOI and superconductivity are intrinsic properties of the materials, the Zeeman splitting is usually generated by applying a rather large magnetic field $[1,2]$, which introduces technical limitations on the use of superconducting elements.

Alternatively, such a spin splitting can be generated without applying an external field by the magnetic proximity effect from a magnetic insulator [21-27]. Indeed, a Zeemanlike splitting at zero magnetic field has been observed in superconducting $\mathrm{Al}$ layers in contact with the ferromagnetic insulator (FI) EuS [28-33]. A recent article reports the first hybrid epitaxial growth of InAs NWs in proximityto EuS and Al [34]. Even though the experiment is inconclusive with regard to Majorana physics, the NWs show signs of a coexisting proximity-induced superconducting gap and spin splitting. These proximitized NWs are pivotal in the study of the topological superconductivity [35-37].

Motivated by this recent experiment [34], we study theoretically a multiband NW in the diffusive regime proximitized by FIs and SCs [see the sketch in Fig. 1(a)]. We show that, apart from the local spin polarization induced by the FI, a nonlocal electronic spin polarization emerges in the NW as a result of an interplay between the magnetic and superconducting proximity effects. The magnetic proximity effect takes place at the FI/NW interface, where the conduction

\footnotetext{
*xianpengzhang@dipc.org

†fs.bergeret@csic.es
}

electrons in the NW interact with the local moments of the FI via spin-exchange coupling. This interaction leads to a Pauli paramagnetic response of the conduction electrons, which is manifested as a locally induced magnetization in the NW at the FI. In addition, the superconducting proximity effect at the NW/SC interface allows for a leakage of Cooper-pair correlations into the NW. The Cooper pairs become polarized by the FI exchange field, admixing to the usual singlet pairing a triplet component of the superconducting correlations. As a result, the Pauli paramagnetic response at the NW/FI interface becomes screened by a spin polarization, which spreads in the NW over large distances, on the order of the superconducting coherence length. This long-ranged component of magnetization is opposite in sign to the Pauli magnetization and its strength is proportional to the condensate density in the NW. In this Rapid Communication, we calculate this nonlocal magnetization as a function of the system parameters, demonstrate its control by the phase difference in a loop geometry, and propose a way of measuring it via spin-dependent spectroscopy.

It is illustrative to review the response of a conventional SC to a Zeeman or exchange field $h(\boldsymbol{r})$ [38-40]. In a normal state, the response is local and leads to a Pauli magnetization $M_{\text {Pauli }}(\boldsymbol{r})=g \mu_{B} v_{F} h(\boldsymbol{r})$ [the dotted black curve in Fig. 1(c)]. Here, $g$ is the $g$-factor, $\mu_{B}$ is the Bohr magneton, and $v_{F}$ is the normal density of states (DOS) at the Fermi level for each spin. When the temperature $T$ is below the critical superconducting temperature, there exists an additional nonlocal contribution to magnetization $M_{\mathrm{NL}}(\boldsymbol{r})$ [dashed red curve in Fig. 1(c)] from the superconducting condensate. In a homogeneous SC at zero temperature, this contribution exactly compensates the Pauli one, $M_{\mathrm{NL}}=-M_{\text {Pauli }}$, for fields $h$ smaller than the superconducting gap $\Delta$. This explains the zero magnetic susceptibility of a SC [41]. In the presence of a spin relaxation (SR), the full magnetization cancellation fails, according to Abrikosov and Gorkov's theory of the Knight 

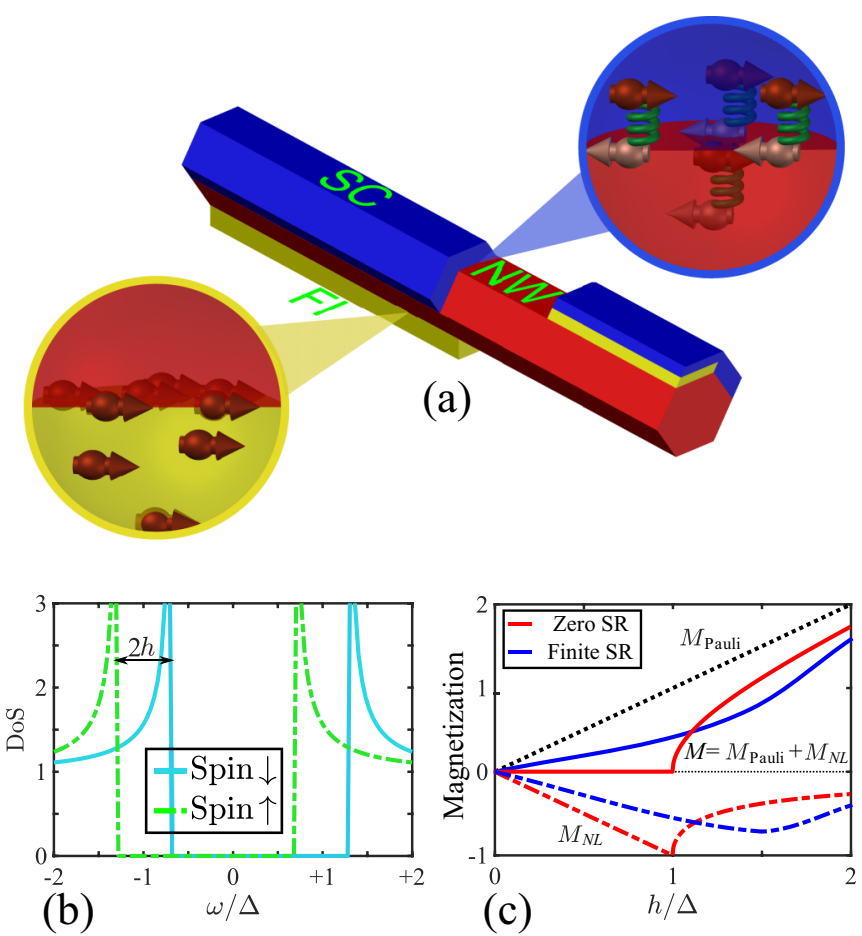

FIG. 1. (a) Sketch of a nanowire (NW) in proximity to superconductors (SCs) and ferromagnetic insulators (FIs). (b) Spin-resolved density of states (DOS) of a spin-split SC. (c) Magnetizations induced in a SC in an homogeneous Zeeman field $h$. The dotted black line describes Pauli magnetization $M_{\text {Pauli }}$ and the solid lines plot the total magnetization $M$ for zero (red) and finite (blue) spin relaxation (SR). The dashed lines show the nonlocal magnetization $M_{\mathrm{NL}}$ given by the difference between $M$ and $M_{\text {Pauli }}$, displayed for zero (red) and finite (blue) SR.

shift in SCs [38]. In Fig. 1(c), we include the SR due to the SOI and static disorder (blue curves). For $h>\Delta$, the compensation is incomplete and the total magnetization reads $M=M_{\text {Pauli }} \sqrt{h^{2}-\Delta^{2}} / h$ [42-44]. One can draw a connection between the nonlocal magnetization and the modified spectrum of the SC [Fig. 1(b)]. The exchange field $h$ leads to both a splitting of the quasiparticle DOS and a reduction of the superconducting gap. As far as the latter is finite, the total magnetization is zero. For $h>\Delta$, the gap closes and a finite magnetization appears as a consequence of an incomplete compensation $\left|M_{\mathrm{NL}}\right|<M_{\text {Pauli }}$. The previous discussion has been introduced for pedagogical purposes, as it is useful when presenting our main results [45].

We now focus on an inhomogeneous system, as shown in Fig. 1(a). It consists of a NW in contact with SCs and FIs. To describe the superconducting proximity effect, we use quasiclassical equations and assume the diffusive regime in the NW. The characteristic length over which the Cooper-pair correlations decay in the $\mathrm{NW}$ is denoted as $\xi_{N}$. To describe the magnetic proximity effect in the FI/NW interface, we follow the approach of Ref. [46] and assume a region of thickness $b$ where the local magnetic moments of FI and the itinerant electrons of NW interact via a spin-exchange coupling. This interaction leads to an interfacial exchange field $h_{\mathrm{ex}}$ acting on the itinerant electrons. Because $b \ll \xi_{N}$, the
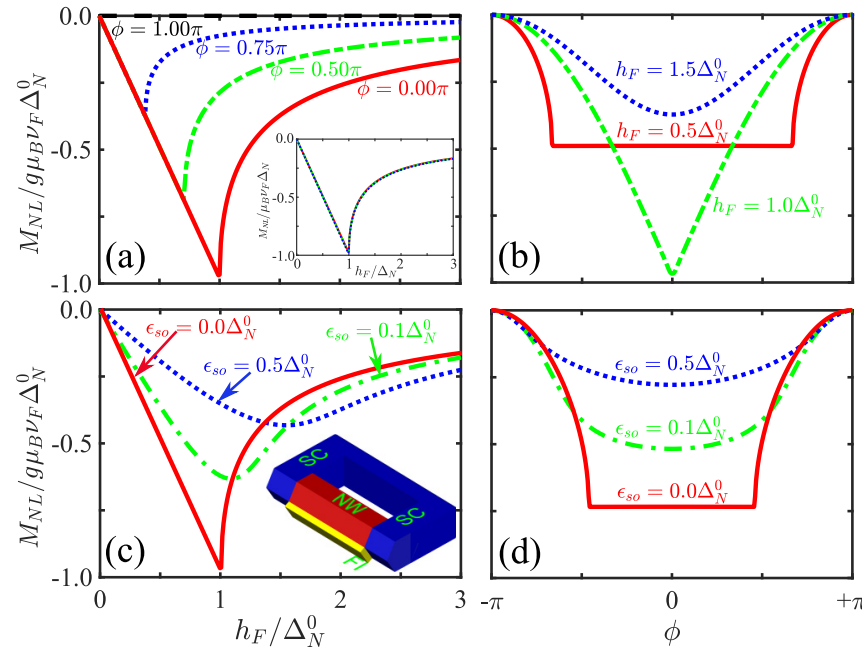

(b)

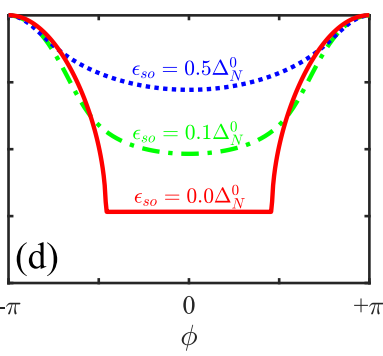

FIG. 2. Nonlocal magnetization $M_{\mathrm{NL}}$ induced in the NW in a $\mathrm{SC} / \mathrm{NW}-\mathrm{FI} / \mathrm{SC}$ setup [see inset of (c)]. (a) and (b) show $M_{\mathrm{NL}}$ as a function of (a) $h_{F} / \Delta_{N}^{0}$ and (b) $\phi$, respectively, in the absence of SR. (c) and (d) show the same dependencies in the presence of SR caused by static disorder and SOI. We have set $\phi=0$ in (c) and $h_{F}=0.75 \Delta_{N}^{0}$ in (d). Other parameters: $T=0$ and $\Delta_{N}^{0}=0.02 \Delta$.

exchange field can be described in the quasiclassical equations by $h_{b}(y)=h_{\mathrm{ex}} b \delta(y)$, where we denote with $y$ the coordinate axis perpendicular to the FI/NW interface [47]. At this stage we can already anticipate the appearance of a nonlocal magnetization in the opposite direction to the one localized at the FI/NW interface. The Cooper pairs in the NW consist of electrons with opposite spins (singlet state). Energetically it is favorable that one electron of the pair with spin parallel to the local exchange localizes at the interface, while the other with opposite spin remains in the NW. Thus, a nonlocal magnetization opposite to the interfacial one is induced in the NW and extends over the characteristic Cooper size $\xi_{N}$. This physical picture resembles the inverse proximity effect in metallic superconductor-ferromagnetic junctions predicted in Refs. [48-50] and experimentally verified in Refs. [51-53].

To quantify this effect we calculate the nonlocal electronic equilibrium spin polarization $M_{\mathrm{NL}}$ induced in the NW. This is given by

$$
\frac{M_{\mathrm{NL}}(X)}{g \mu_{B} v_{F}}=\frac{1}{2} \int_{-\infty}^{+\infty} d \omega f(\omega)\left[N^{\uparrow}(\omega, X)-N^{\downarrow}(\omega, X)\right],
$$

where $f(\omega)=1 /\left(e^{\omega / T}+1\right)$ is the equilibrium Fermi distribution function, and $N^{\uparrow / \downarrow}(\omega, X)$ are the local DOS for spin-up and spin-down electrons. The exchange field at the FI/NW leads to $N^{\uparrow} \neq N^{\downarrow}$ and hence to a finite $M_{\mathrm{NL}}$. In addition to the nonlocal term, there is the Pauli magnetization localized at the FI/NW interface $M_{\text {Pauli }}=g \mu_{B} v_{F} h_{\mathrm{ex}} b \delta(y)$. Thus, the total magnetization equals $M_{\text {Pauli }}+M_{\mathrm{NL}}$.

We consider first the SC/NW-FI/SC setup sketched in the inset of Fig. 2(c). The NW is in contact with a FI, and sandwiched between two SCs. The phase difference between the SCs, $\phi$, can be tuned by a magnetic flux, when the junction is part of a superconducting loop. We assume a diffusive NW in order to use the well-established Usadel equation [54]. In this respect, our results apply straightforwardly to a metallic 
NW such as $\mathrm{Cu}$. In semiconducting NWs, the degree of disorder depends on doping. For example, the InAs wires studied in the experiments of Refs. [55-58] are in a metallic regime and are good candidates for the verification of our predictions. We denote with $x$ the axis of the NW of length $L_{N}$. The NW-FI interface is orthogonal to the $y$ axis and the NW width in this direction is $W_{N}$. In this first example we assume that $W_{N}, L_{N} \ll \xi_{N}$ and integrate the quasiclassical equations over the volume of the NW. The integration in the $y$ direction results in an effective exchange field $h_{F}=h_{\mathrm{ex}} b / W_{N}$, whereas the integration over $x$ can be performed with the help of the Kupriyanov-Lukichev boundary conditions [59] and accounts for the superconducting proximity effect. In this way we obtain a compact expression for the DOS [60],

$$
N^{\eta}(\omega)=\left|\operatorname{Re}\left\{\frac{\omega_{r}+\eta h_{F}}{\sqrt{\left(\omega_{r}+\eta h_{F}\right)^{2}-\left(\Delta_{r}\right)^{2}}}\right\}\right|,
$$

where $\eta= \pm 1$ for spin $\uparrow / \downarrow$. This expression has the same structure as the BCS DOS of a spin-split superconductor with renormalized frequency $\omega_{r}=\omega+2 i \epsilon_{b} \mathcal{G}_{S}$ and order parameter $\Delta_{r}=2 \epsilon_{b} \cos (\phi / 2) \mathcal{F}_{S}$, where $\mathcal{G}_{S}=-i \omega / \sqrt{\Delta^{2}-\omega^{2}}$, $\mathcal{F}_{S}=\Delta / \sqrt{\Delta^{2}-\omega^{2}} . \epsilon_{b}=D /\left(L_{N} \sigma_{N} R_{\square}\right)$ is an energy proportional to the tunneling rate across the $\mathrm{NW} / \mathrm{SC}$ interface, where $R_{\square}$ is the interface resistance per area, $D$ is the diffusion coefficient, and $\sigma_{N}$ is the conductivity of the NW. Equation (2) is the generalization of the short-junction limit expression for the DOS [61-63] in the presence of a FI. With its help we provide below a clear physical picture of the main effect by making a connection between the spectrum of the junction and the spectral properties of the bulk system.

From Eq. (2), one can calculate the gap induced in the NW by the superconducting proximity effect. In the limit of transparent contact, $\epsilon_{b} \gg \Delta$, this gap is of the same order as the SC gap and the spin splitting is negligibly small. In the case of a finite NW/SC barrier, when $\epsilon_{b} \ll \Delta$, Eq. (2) describes a NW with an induced minigap, $\Delta_{N}=\Delta_{N}^{0} \cos (\phi / 2)$, with $\Delta_{N}^{0}=2 \epsilon_{b}$, and a spin splitting in the DOS due to the effective exchange field $h_{F}$. In all cases the minigap induced in the NW is maximum when $\phi=0$ and vanishes at $\phi=\pi$. By substituting Eq. (2) into Eq. (1), we obtain the nonlocal magnetization $M_{\mathrm{NL}}$ plotted in Fig. 2. As far as $h_{F}<\Delta_{N}$, nonlocal magnetic moments $M_{\mathrm{NL}} W_{N} A$ compensate the Pauli ones, $\int_{b} M_{\text {Pauli }}=g \mu_{B} v_{F} h_{\text {ex }} b A$, localized at the FI/NW interface, with $A$ being the area of the FI/NW interface. At $h_{F}=\Delta_{N}, M_{\mathrm{NL}}$ reaches a maximum value, $g \mu_{B} v_{F} \Delta_{N}$, and decays as $h_{F}-\sqrt{h_{F}^{2}-\Delta_{N}^{2}}$ for $h_{F}>\Delta_{N}$ [42-44]. This is the same behavior as the bulk superconductor discussed in Fig. 1(c), after identifying $\Delta$ and $h$ with the induced minigap $\Delta_{N}$ and effective exchange field $h_{F}$, respectively. This analogy is clearly seen if we plot the curves of Fig. 2(a) as a function $h_{F} / \Delta_{N}$. In this case all curves collapse into one [inset of Fig. 2(a)] coinciding with the behavior shown in Fig. 1(c). In Fig. 2(b) we show the dependence of $M_{\mathrm{NL}}$ on the phase difference $\phi$ for different values of $h_{F}$. When $h_{F} \leqslant \Delta_{N}^{0}, M_{\mathrm{NL}}$ remains constant for all phases smaller than $\arccos h_{F} / \Delta_{N}^{0}$ [red curve in Fig. 2(b)]. In other words, as far as $h_{F}$ is smaller than the induced gap $\Delta_{N}=\Delta_{N}^{0} \cos (\phi / 2)$, the $M_{\mathrm{NL}}(\phi)$ curve shows a plateau at the value opposite to $M_{\text {Pauli }}$. Interestingly, the value of $M_{\mathrm{NL}}$ is proportional to the distance between the
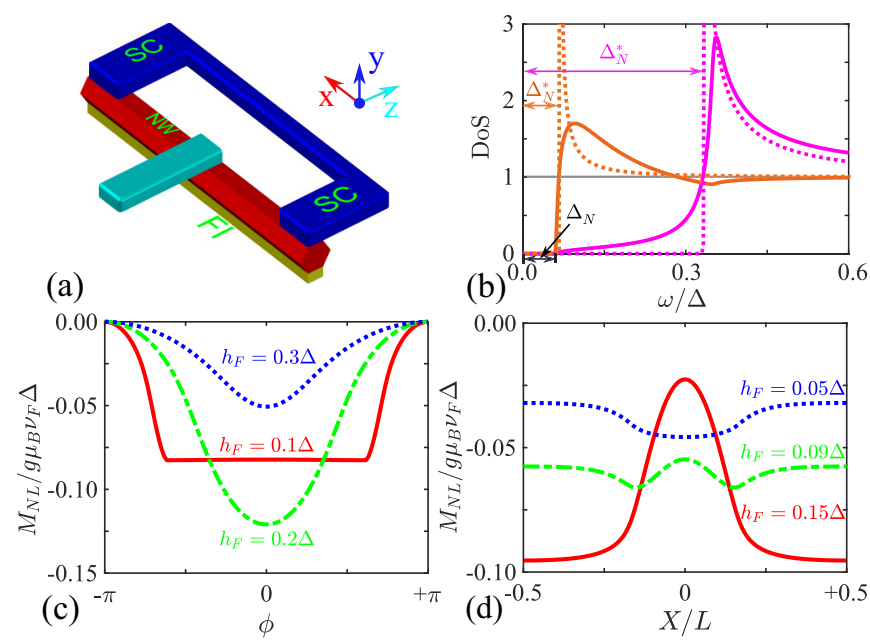

FIG. 3. (a) Sketch of a SC-FI-SC NW structure with a tunneling probe (bright blue). (b) DOS of the NW with $L=4.7 \xi_{0}$. Here, the orange and magenta curves correspond to DOS at the center $(X=$ $\left.L_{N} / 2\right)$ and the end $\left(X=L_{N} / 6\right)$ of the NW, respectively. The dotted lines show the BCS-like DOS with a gap equal to $\Delta^{*}(X)$. The latter is defined by the intersection point between the actual DOS and the one in the normal state. (c), (d) Nonlocal magnetization $M_{\mathrm{NL}}$ induced in the NW, as a function of (c) phase difference $\phi$ and (d) position $X$. We have set $L=2.1 \xi_{0}$ and $X=0$ in (c), while $L=4.7 \xi_{0}$ and $\phi=0$ in (b) and (d). In all panels, other parameters are chosen as follows: $T=0, \epsilon_{s o}=0, \epsilon_{b}=\Delta / 2, \xi_{0}=\sqrt{D / \Delta}$, and $L_{S} / L_{N}=1 / 3$.

coherent peaks in the spin-splitting DOS, similar to those shown in Fig. 1(b). Indeed, in the present case when $\Delta_{N} \ll \Delta$, according to Eq. (2), the peaks at positive energies occur at $\omega^{\uparrow, \downarrow} \approx \Delta_{N}(\phi) \pm h_{F}$ [60]. The maximum modulation is achieved for $h_{F}=\Delta_{N}^{0}$ [green curve in Fig. 2(b)] in which the full screening of $M_{\mathrm{NL}}$ only occurs at $\phi=0$. For larger values of $h_{F}$, the NW is gapless and $M_{\mathrm{NL}}(\phi)$ is overall reduced (blue curve).

In the presence of SOI, electron spin channels are mixed. In this case the DOS of the NW is described by Eq. (2), after replacing $\omega_{r}$ and $\Delta_{r}$ by $\omega_{r}^{\eta}=\omega+i \Delta_{N}^{0} \mathcal{G}_{S}+2 i \epsilon_{s o} G_{N}^{-\eta}$ and $\Delta_{r}^{\eta}=\Delta_{N} \mathcal{F}_{S}+2 \epsilon_{s o} F_{N}^{-\eta}$, respectively. Here, $F_{N}^{\eta}$ and $G_{N}^{\eta}$ are the normal and anomalous parts of the retarded Green's function of the NW, respectively [60]. $\epsilon_{s o}$ is the spin-relaxation rate due to SOI. The effect of a finite SR is shown in Figs. 2(c) and 2(d). As expected from the analogy with the bulk SC, Fig. 1(c), the main effect of the SR is the uncompensated screening of the Pauli magnetization, $-M_{N L}<M_{\text {Pauli }}$, as shown by the green and blue curves in Fig. 2(c). In addition, the SR leads to a shift of the maximum of the $M_{\mathrm{NL}}\left(h_{F}\right)$ curves towards larger values of $h_{F}$, such that, for $h_{F}>\Delta_{N}, M_{\mathrm{NL}}$ is enhanced by the SR. This is due to the reduction of the effective exchange field [64], which results into the right shift of $M_{\mathrm{NL}}$ with respect to $h_{F}$ in analogy with the bulk case shown by the dotted-dashed blue curve of Fig. 1(c).

So far we have analyzed a short NW sandwiched between two SCs. In a more realistic setup, the length of the NW, $L_{N}$, can be larger than the $\xi_{N}$. Moreover, in typical lateral structures the NW is partially covered by SC films of length $L_{S}$. Such a lateral setup is sketched in Fig. 3(a). We assume that the NW is grown on top of a FI substrate, and that its 
cross-section dimensions are smaller than $\xi_{N}$. In this case one can integrate the Usadel equation over the cross section and reduce the problem to an effective one-dimensional (1D) geometry (details are given in the Supplemental Material [60]). Hereafter, we assume a symmetric setup with $L_{S}=L_{N} / 3$ and $L_{F}=L_{N}$ (other situations are analyzed in the Supplemental Material [60]), such that the distance between the SCs is $L=L_{N} / 3$, and solve the Usadel equation numerically. We neglect the effect of SOI. This is a good approximation if the $\mathrm{NM}$ is a metal such as $\mathrm{Cu}$, for which the SR rate is much smaller than the gap [65]. But also in InAs, the typical SR time is $\tau_{s} \simeq 0.02-1.00 \mathrm{~ns}$ [66-68], which corresponds to $\epsilon_{s o}=\hbar / \tau_{s} \simeq 1-30 \mu \mathrm{eV}$, whereas the induced gap may reach $150 \mu \mathrm{eV}$ or even larger [12,69], such that the ratio $\epsilon_{s o} / \Delta<1$.

Once induced, the minigap is constant in all the NW [70]. Its value depends on the distance between the superconducting electrodes and the characteristic barrier energy $\epsilon_{b}=$ $D /\left(W_{N} R_{\square} \sigma_{N}\right)$. In the short limit, $L_{N} \ll \xi_{N}, M_{\mathrm{NL}}$ is almost constant in the NW and the results are similar to those shown in Figs. 2(a) and 2(b) [60]. More interesting is the case when $L_{N}$ is of the order of $\xi_{N}$. Numerical results of the spatial dependence $M_{\mathrm{NL}}(X)$ for $L_{N}=4.7 \xi_{0}$ and different values of $h_{F}$ are shown in Fig. 3(d). Remarkably, the shape of the $M_{\mathrm{NL}}(X)$ curve depends on the strength of $h_{F}$. These different behaviors can be explained in light of Eq. (1). The integrand in this expression can be well approximated by replacing the exact DOS, $N(\omega, X)$ by a BCS-like one, $N_{\mathrm{BCS}}\left[\omega, \Delta_{N}^{*}(X)\right]$, with a position-dependent pseudogap $\Delta_{N}^{*}(X)$ defined as the energy where $N(\omega)$ intersects with the one in the normal state $N_{0}(\omega)=1$, as shown in Fig. 3(b). Whereas the real minigap $\Delta_{N}$ is position independent, $\Delta_{N}^{*}$ is not. In fact, the pseudogap is smaller in the middle of the wire, becoming larger in the regions below the SCs [see also Fig. 2(d) in the Supplemental Material [60]]. The shape of the $M_{\mathrm{NL}}(X)$ is determined by the ratio $h_{F} / \Delta_{N}^{*}(X)$ in the same way as in the short-junction limit $h_{F} / \Delta_{N}^{0}$ determines $M_{N}$ [see Figs. 2(a) and 2(c)]. Indeed, for a given $h_{F}$ with $h_{F}<\Delta_{N}^{*}(X)$ for all $X$, the values of $\left|M_{\mathrm{NL}}\right|$ increase towards the middle of the wire [blue curve in Fig. 3(d)]. In contrast, if $\Delta^{*}(-L / 2)>$ $h_{F}>\Delta^{*}(0)$, then a double-minima curve is obtained (green curve). Larger values of $h_{F}$ lead to $\left|M_{N}(X)\right|$ with a minimum at $X=0$ (red curve). The actual shape of the curve can be inferred from the $X$ dependence of $\Delta^{*}$ which is shown in Fig. 2(c) in the Supplemental Material [60]. Finally, Fig. 3(c) shows the phase dependence of $M_{\mathrm{NL}}$ calculated in the center of the wire for different values of $h_{F}$. The result at low temperatures is qualitatively similar to the one obtained for the simpler setup analyzed in Fig. 2(b): For values of $h_{F}$ smaller than the pseudogap $\Delta_{N}^{*}, M_{\mathrm{NL}}(\phi)$ remains almost constant up to the value of $\varphi$ for which $\Delta_{N}^{*}(\phi)=h_{F}$ [red curve in Fig. 3(c)].

Finally, we discuss the possible ways of detecting $M_{\mathrm{NL}}$ via its dependence on the phase difference in a Josephson junction geometry. As discussed above, the magnetic moment $M_{\mathrm{NL}}$ depends crucially on the spectral properties of the proximitized NW, which in turn can be controlled by tuning the phase difference. This has been demonstrated experimentally in spectroscopy measurements, for example, by using a superconducting quantum interference proximity transistor (SQUIPT) [71-74], sketched in Fig. 3(a), or by combining scanning tunneling microscopy/atomic force microscopy (STM/AFM) techniques [70]. In these experiments the phase difference, and hence the minigap, is controlled by the magnetic flux through a superconducting the loop [75,76]. In the present case the wire is in contact with a FI, and hence the DOS in the NW is spin split due to the exchange field at the FI/NM interface. This should manifest as a splitting of the peaks at the edge of the gap. According to our predictions, if the SR is negligibly small, the observed splitting of the peaks remains almost constant, as far as the phase-dependent pseudogap $\Delta_{N}^{*}$ is larger than the effective exchange field [see red curves in Figs. 2(b) and 3(c)]. The splitting in the DOS of the NW can be detected by measuring the differential conductance with a tunneling probe attached to the NW, as shown in Fig. 3(a). When the phase difference is larger than $\arccos \left(h_{F} / \Delta_{N}^{0}\right)$, then we predict a rapid suppression of the splitting as the phase difference is further increased. The results of Fig. 3 are obtained when SOI is negligible. If it is not, the all sharp features will vanish, and the red curve in Fig. 3(c) will be modified similarly to those in Fig. 2(d) when increasing $\epsilon_{s o}$. It is also interesting to note that the tuning of minigap with the phase difference can lead to a phase-tuned topological superconductivity [77]. Moreover, a comparison of experimental results with the curves in Figs. 2(b) and 3(c) may provide useful information about the proximity-induced gap and field in the NW.

A more direct measurement of $M_{\mathrm{NL}}$ and its phase dependence can be achieved by using a ferromagnetic probe, tunnel-coupled to NW, as shown in Fig. 3(a). We assume that the polarizations of the probe and the FI can be tuned between parallel (P) and antiparallel (AP) configurations. The measured differential conductance at low temperature is proportional to the DOS in the NW. In particular, the difference between the conductances in the $\mathrm{P}$ and AP configurations is proportional to the spectral magnetization induced in the NW. Namely, $G_{P}(V)-G_{A P}(V)=p G_{0}\left[N_{\uparrow}(V)-N_{\downarrow}(V)\right]$, where $p$ is the polarization of the probe/NW tunnel junction and $G_{0}$ is normal-state tunneling conductance. The total induced magnetization can then be obtained from Eq. (1) by knowing the normal-state properties of the tunneling contact. By using the SQUIPT setup of Fig. 3(a), one can tune the phase difference by an external magnetic field and measure the $N_{\mathrm{NL}}(\phi)$ curve. From a material perspective, our theoretical description is based on the diffusive approach and therefore our findings can be best verified in metallic $\mathrm{NM}$, as $\mathrm{Cu}$, or highly doped semiconducting nanowires, as those used in Refs. [55-58]. For the FI, EuS is the best candidate. Interfacial exchange fields of the order of tens of tesla has been reported in a system combing EuS with metals and graphene [26,31] which would lead to effective $h_{F} \sim 10^{-2}-10^{-1} \mathrm{meV}$ such that one can reach all regimes studied above. Moreover, the strength of the effective exchange field can be tuned by an external magnetic field [78].

In conclusion, we predict the appearance of a nonlocal magnetization $M_{\mathrm{NL}}$ in a NW when proximitized to SCs and a FI. This magnetization appears as a consequence of the interplay between the long-range superconducting correlations induced in the NW and the exchange field localized at the FI/NW interface. The sign of $M_{\mathrm{NL}}$ is opposite to the 
local Pauli spin polarization right at the FI/NW interface and its value can be controlled by the phase difference between superconducting electrodes in a Josephson junction setup.
This work was supported by Spanish Ministerio de Ciencia e Innovacion (MICINN) through the Project FIS2017-82804P, and EU's Horizon 2020 research and innovation program under Grant Agreement No. 800923 (SUPERTED).
[1] R. M. Lutchyn, J. D. Sau, and S. Das Sarma, Phys. Rev. Lett. 105, 077001 (2010).

[2] Y. Oreg, G. Refael, and F. von Oppen, Phys. Rev. Lett. 105, 177002 (2010).

[3] V. Mourik, K. Zuo, S. M. Frolov, S. Plissard, E. P. Bakkers, and L. P. Kouwenhoven, Science 336, 1003 (2012).

[4] L. P. Rokhinson, X. Liu, and J. K. Furdyna, Nat. Phys. 8, 795 (2012).

[5] A. Das, Y. Ronen, Y. Most, Y. Oreg, M. Heiblum, and H. Shtrikman, Nat. Phys. 8, 887 (2012).

[6] A. D. K. Finck, D. J. Van Harlingen, P. K. Mohseni, K. Jung, and X. Li, Phys. Rev. Lett. 110, 126406 (2013).

[7] S. M. Albrecht, A. P. Higginbotham, M. Madsen, F. Kuemmeth, T. S. Jespersen, J. Nygård, P. Krogstrup, and C. Marcus, Nature (London) 531, 206 (2016).

[8] M. Deng, S. Vaitiekenas, E. B. Hansen, J. Danon, M. Leijnse, K. Flensberg, J. Nygård, P. Krogstrup, and C. M. Marcus, Science 354, 1557 (2016).

[9] H. J. Suominen, M. Kjaergaard, A. R. Hamilton, J. Shabani, C. J. Palmstrøm, C. M. Marcus, and F. Nichele, Phys. Rev. Lett. 119, 176805 (2017).

[10] F. Nichele, A. C. C. Drachmann, A. M. Whiticar, E. C. T. O'Farrell, H. J. Suominen, A. Fornieri, T. Wang, G. C. Gardner, C. Thomas, A. T. Hatke, P. Krogstrup, M. J. Manfra, K. Flensberg, and C. M. Marcus, Phys. Rev. Lett. 119, 136803 (2017).

[11] S. Takei, B. M. Fregoso, H.-Y. Hui, A. M. Lobos, and S. Das Sarma, Phys. Rev. Lett. 110, 186803 (2013).

[12] W. Chang, S. Albrecht, T. Jespersen, F. Kuemmeth, P. Krogstrup, J. Nygård, and C. M. Marcus, Nat. Nanotechnol. 10, 232 (2015).

[13] R. M. Lutchyn, T. D. Stanescu, and S. Das Sarma, Phys. Rev. Lett. 106, 127001 (2011).

[14] X.-L. Qi and S.-C. Zhang, Rev. Mod. Phys. 83, 1057 (2011).

[15] S. R. Elliott and M. Franz, Rev. Mod. Phys. 87, 137 (2015).

[16] C. Beenakker, Annu. Rev. Condens. Matter Phys. 4, 113 (2013).

[17] J. Alicea, Rep. Prog. Phys. 75, 076501 (2012).

[18] R. t. Lutchyn, E. Bakkers, L. P. Kouwenhoven, P. Krogstrup, C. Marcus, and Y. Oreg, Nat. Rev. Mater. 3, 52 (2018).

[19] S. D. Sarma, M. Freedman, and C. Nayak, npj Quantum Inf. 1, 15001 (2015).

[20] T. D. Stanescu and S. Tewari, J. Phys.: Condens. Matter 25, 233201 (2013).

[21] F. S. Bergeret, M. Silaev, P. Virtanen, and T. T. Heikkilä, Rev. Mod. Phys. 90, 041001 (2018).

[22] F. Giazotto and F. Taddei, Phys. Rev. B 77, 132501 (2008).

[23] H.-X. Yang, A. Hallal, D. Terrade, X. Waintal, S. Roche, and M. Chshiev, Phys. Rev. Lett. 110, 046603 (2013).

[24] S. V. Eremeev, V. N. Men'shov, V. V. Tugushev, P. M. Echenique, and E. V. Chulkov, Phys. Rev. B 88, 144430 (2013).

[25] P. Virtanen, F. S. Bergeret, E. Strambini, F. Giazotto, and A. Braggio, Phys. Rev. B 98, 020501(R) (2018).
[26] P. Wei, S. Lee, F. Lemaitre, L. Pinel, D. Cutaia, W. Cha, F. Katmis, Y. Zhu, D. Heiman, J. Hone et al., Nat. Mater. 15, 711 (2016).

[27] F. Katmis, V. Lauter, F. S. Nogueira, B. A. Assaf, M. E. Jamer, P. Wei, B. Satpati, J. W. Freeland, I. Eremin, D. Heiman et al., Nature (London) 533, 513 (2016).

[28] X. Hao, J. S. Moodera, and R. Meservey, Phys. Rev. Lett. 67, 1342 (1991).

[29] R. Meservey, P. Tedrow, and P. Fulde, Phys. Rev. Lett. 25, 1270 (1970).

[30] X. Hao, J. S. Moodera, and R. Meservey, Phys. Rev. B 42, 8235 (1990).

[31] E. Strambini, V. N. Golovach, G. De Simoni, J. S. Moodera, F. S. Bergeret, and F. Giazotto, Phys. Rev. Materials 1, 054402 (2017).

[32] J. S. Moodera, X. Hao, G. A. Gibson, and R. Meservey, Phys. Rev. Lett. 61, 637 (1988).

[33] M. Rouco, S. Chakraborty, F. Aikebaier, V. N. Golovach, E. Strambini, J. S. Moodera, F. Giazotto, T. T. Heikkilä, and F. S. Bergeret, Phys. Rev. B 100, 184501 (2019).

[34] Y. Liu, S. Vaitiekenas, S. Martí-Sánchez, C. Koch, S. Hart, Z. Cui, T. Kanne, S. A. Khan, R. Tanta, S. Upadhyay et al., Nano Lett. 20, 456 (2019).

[35] J. D. Sau, R. M. Lutchyn, S. Tewari, and S. Das Sarma, Phys. Rev. Lett. 104, 040502 (2010).

[36] S.-P. Lee, J. Alicea, and G. Refael, Phys. Rev. Lett. 109, 126403 (2012).

[37] G. Livanas, M. Sigrist, and G. Varelogiannis, Sci. Rep. 9, 1 (2019).

[38] A. A. Abrikosov and L. P. Gorkov, J. Exp. Theor. Phys. 15, 752 (1962).

[39] A. Larkin and A. Varlamov, Theory of Fluctuations in Superconductors (Clarendon, Oxford, U.K., 2005).

[40] P. Fulde and R. A. Ferrell, Phys. Rev. 135, A550 (1964).

[41] K. Yosida, Phys. Rev. 110, 769 (1958).

[42] F. S. Bergeret, A. F. Volkov, and K. B. Efetov, Rev. Mod. Phys. 77, 1321 (2005).

[43] N. I. Karchev, K. B. Blagoev, K. S. Bedell, and P. B. Littlewood, Phys. Rev. Lett. 86, 846 (2001).

[44] R. Shen, Z. M. Zheng, S. Liu, and D. Y. Xing, Phys. Rev. B 67, 024514 (2003).

[45] Strictly speaking, for a large enough field $h$, the superconducting gap has to be determined self-consistently, and an inhomogeneous superconducting phase may appear $[39,40]$. The situation is simpler when superconductivity is induced in a nonsuperconducting material via the proximity effect. In this case the self-consistency is not needed and the exchange field can be arbitrarily large. This is the case considered in the rest of this Rapid Communication.

[46] X.-P. Zhang, F. S. Bergeret, and V. N. Golovach, Nano Lett. 19, 6330 (2019). 
[47] F. S. Bergeret, K. B. Efetov, and A. I. Larkin, Phys. Rev. B 62, 11872 (2000).

[48] F. S. Bergeret, A. F. Volkov, and K. B. Efetov, Phys. Rev. B 69, 174504 (2004).

[49] F. Bergeret, A. Volkov, and K. Efetov, Europhys. Lett. 66, 111 (2004).

[50] S. M. Dahir, A. F. Volkov, and I. M. Eremin, Phys. Rev. B 100, 134513 (2019).

[51] J. Xia, V. Shelukhin, M. Karpovski, A. Kapitulnik, and A. Palevski, Phys. Rev. Lett. 102, 087004 (2009).

[52] R. I. Salikhov, I. A. Garifullin, N. N. Garifyanov, L. R. Tagirov, K. Theis-Bröhl, K. Westerholt, and H. Zabel, Phys. Rev. Lett. 102, 087003 (2009).

[53] R. I. Salikhov, N. N. Garifyanov, I. A. Garifullin, L. R. Tagirov, K. Westerholt, and H. Zabel, Phys. Rev. B 80, 214523 (2009).

[54] K. D. Usadel, Phys. Rev. Lett. 25, 507 (1970).

[55] F. Giazotto, P. Spathis, S. Roddaro, S. Biswas, F. Taddei, M. Governale, and L. Sorba, Nat. Phys. 7, 857 (2011).

[56] J. Tiira, E. Strambini, M. Amado, S. Roddaro, P. San-Jose, R. Aguado, F. Bergeret, D. Ercolani, L. Sorba, and F. Giazotto, Nat. Commun. 8, 1 (2017).

[57] A. Iorio, M. Rocci, L. Bours, M. Carrega, V. Zannier, L. Sorba, S. Roddaro, F. Giazotto, and E. Strambini, Nano Lett. 19, 652 (2018).

[58] E. Strambini, A. Iorio, O. Durante, R. Citro, C. Sanz-Fernández, C. Guarcello, I. Tokatly, A. Braggio, M. Rocci, N. Ligato et al., arXiv:2001.03393.

[59] M. Y. Kupriyanov and V. F. Lukichev, J. Exp. Theor. Phys. 67, 1163 (1988)

[60] See Supplemental Material at http://link.aps.org/supplemental/ 10.1103/PhysRevB.101.180502 for the derivation of Usadel equations which includes Refs. [38-40,42-44,46,47,59,79].

[61] R. Seviour and A. F. Volkov, Phys. Rev. B 61, R9273 (2000).

[62] J. Börlin, W. Belzig, and C. Bruder, Phys. Rev. Lett. 88, 197001 (2002).

[63] E. V. Bezuglyi, E. N. Bratus, and V. S. Shumeiko, Phys. Rev. B 83, 184517 (2011).
[64] In fact, in the limit $\epsilon_{s o} \ll h_{F}, \Delta_{N}$, the effective exchange field acting on the conducting electrons becomes $h_{\mathrm{eff}} \simeq h_{F}\left[1-2 \epsilon_{s o}^{2} /\left(h_{F}^{2}-\Delta_{N}^{2}\right)\right]$ for $h_{F}>\Delta_{N}$ and $h_{\mathrm{eff}} \simeq h_{F}[1-$ $\left.\epsilon_{s o} / \sqrt{h_{F}\left(\Delta_{N}-h_{F}\right)}\right]$ for $h_{F}<\Delta_{N}[60]$.

[65] E. Villamor, M. Isasa, L. E. Hueso, and F. Casanova, Phys. Rev. B 87, 094417 (2013)

[66] P. Murzyn, C. Pidgeon, P. Phillips, M. Merrick, K. Litvinenko, J. Allam, B. Murdin, T. Ashley, J. Jefferson, A. Miller et al., Appl. Phys. Lett. 83, 5220 (2003).

[67] P. H. Song and K. W. Kim, Phys. Rev. B 66, 035207 (2002).

[68] B. N. Murdin, K. Litvinenko, J. Allam, C. R. Pidgeon, M. Bird, K. Morrison, T. Zhang, S. Clowes, W. R. Branford, J. Harris, and L. F. Cohen, Phys. Rev. B 72, 085346 (2005).

[69] M. Kjærgaard, F. Nichele, H. J. Suominen, M. P. Nowak, M. Wimmer, A. R. Akhmerov, J. A. Folk, K. Flensberg, J. Shabani, C. J. Palmstrøm et al., Nat. Commun. 7, 1 (2016).

[70] H. le Sueur, P. Joyez, H. Pothier, C. Urbina, and D. Esteve, Phys. Rev. Lett. 100, 197002 (2008).

[71] F. Giazotto, J. T. Peltonen, M. Meschke, and J. P. Pekola, Nat. Phys. 6, 254 (2010).

[72] M. Meschke, J. T. Peltonen, J. P. Pekola, and F. Giazotto, Phys. Rev. B 84, 214514 (2011).

[73] F. Giazotto and F. Taddei, Phys. Rev. B 84, 214502 (2011).

[74] A. Ronzani, C. Altimiras, and F. Giazotto, Phys. Rev. Applied 2, 024005 (2014)

[75] E. Strambini, S. D’Ambrosio, F. Vischi, F. Bergeret, Y. V. Nazarov, and F. Giazotto, Nat. Nanotechnol. 11, 1055 (2016).

[76] A. Ronzani, S. D'Ambrosio, P. Virtanen, F. Giazotto, and C. Altimiras, Phys. Rev. B 96, 214517 (2017).

[77] A. Fornieri, A. M. Whiticar, F. Setiawan, E. Portolés, A. C. Drachmann, A. Keselman, S. Gronin, C. Thomas, T. Wang, R. Kallaher et al., Nature (London) 569, 89 (2019).

[78] Y. M. Xiong, S. Stadler, P. W. Adams, and G. Catelani, Phys. Rev. Lett. 106, 247001 (2011).

[79] J. C. Hammer, J. C. Cuevas, F. S. Bergeret, and W. Belzig, Phys. Rev. B 76, 064514 (2007). 